International Journal of Computer Networks \& Communications (IJCNC) Vol.3, No.5, Sep 2011

\title{
Performance Of Iterated EKF Technique To Estimate TIME VARYING CHANNEL UsING Pilot Assisted METHOd IN MIMO-OFDM SYSTEM
}

\author{
Rajendra Prasad.K ${ }^{1}$, Srinivasan.M ${ }^{2}$, Sravya.P ${ }^{3}$, Gnaneswari.P ${ }^{4}$, \\ Naga Harshavardhan. $\mathrm{K}^{4}$, Pranob K Charles ${ }^{5}$ \\ ${ }^{1}$ Asst. Professor, School of Electrical Sciences, KL University, A.P, India \\ ${ }^{2}$ Professor, Meerut Institute of Engineering and Technology, Meerut, U.P, India \\ ${ }^{3}$ PG student, SBIT, Khammam, A.P, India \\ ${ }^{4}$ Student Members, Signal Processing Research Group, KL University, A.P, India \\ ${ }^{5}$ Assoc. Professor, School of Electrical Sciences, KL University, A.P, India \\ krajendraec@gmail.com
}

\begin{abstract}
In this paper Iterative Extended Kalman Filter (IEKF) technique has been proposed to estimate the time varying channel for multiple-input multiple-output (MIMO) orthogonal frequency division multiplexing systems (OFDM). Channel state information (CSI) plays major role to improve the performance of any wireless communication system for different fading channels to detect the data. Kalman Filter $(K F)$ is most powerful technique for linear processing and it is more suitable to estimate additive white gaussian noise (AWGN) channels. But it is not fit for non-linear problems since wireless channels have some nonlinear characteristics. In this paper, IEKF technique has been proposed to estimate time varying channel, and comparative analysis has been done with the techniques which are proposed based on LS, MMSE and EKF. Simulations also demonstrated that, channel estimation based on IEKF having significant improvement in aspect of bit error rate (BER) and mean square error (MSE) with modest computational complexity.
\end{abstract}

\section{KEYWORDS}

MIMO-OFDM, Channel estimation, Iterative Extended Kalman Filter, Mean Square Error

\section{INTRODUCTION}

In Point-to-Point wireless links, multiple-input multiple-output (MIMO) systems that utilize multiple antennas at both transmitter and receiver, have demonstrated the potential for increased line capacity and diversity gain in rich multipath environments similar to cellular mobile and wireless broadband communication systems compared to conventional single-input single-output (SISO), single-input multiple -output (SIMO), and multiple-input single-output (MISO) systems. The advantages originate from the multiple spatial channels, which are provided by the multiple antennas together with the scattering environment surrounding the transmitters and the receivers. MIMO technology [1-2] offers significant increases in data throughput and link range without additional bandwidth or transmit power. It is remarkable that 
International Journal of Computer Networks \& Communications (IJCNC) Vol.3, No.5, Sep 2011

the maximum achievable diversity gain of a MIMO system with $\mathrm{N}_{\mathrm{T}}$ antenna elements at the transmitter and $N_{R}$ antenna elements at the receiver is $N_{T} \times N_{R}$.

Since in wireless communication systems, channel response is time variant, channel estimation is an essential part of the receiver. And space-time coding technique [3-4] is often applied to MIMO systems to mitigate channel fading without sacrificing bandwidth and becoming attractive in broadband wireless systems. A system that combines MIMO system, space-time coding and OFDM can provide spectral efficiency and higher data transmission over a fading channel. In order to attain the advantages of MIMO systems [5], it is necessary that the receiver and/or transmitter have access of channel state information (CSI).Hence effective channel estimation algorithms are needed to guarantee the performance of communication. Several classes of methods are available to estimate the channel response among them which includes Pilot-Aided or Training-Based Channel Estimation (TBCE) [6-7] schemes relying on training sequences that are known a priori to the receiver are multiplexed along with the data stream for channel estimation. Blind Channel Estimation (BCE) methods identifying channel only from the received sequences, it is carried out by evaluating the statistical information of the channel and certain properties of the transmitted signals. Blind Channel Estimation has its advantage in that it has no overhead loss; it is only applicable to slowly time-varying channels due to its need for a long data record. Semi-Blind Channel Estimation (SBCE) approaches as a combination of blind and training technique, utilizing pilots and other natural constraints to perform channel estimation, and Decision Directed channel Estimation (DDCE) could be viewed as Pilot-Aided Channel Estimation scheme that is employing approximately hundred percent pilot information symbols for channel estimation in comparison with the purely pilot-aided scheme with sparse available pilot symbols for the same estimation.

One of the most common approaches to identify MIMO CSI is TBCE. Channel estimates are often achieved by multiplexing known symbols, so called pilot symbols into data sequence, and this technique is called Pilot Symbol Aided Modulation (PSAM). This class of estimation is attractive, especially when it decouples symbol detection from channel estimation, and thus simplifies the receiver implementation and slows down the required identification conditions. In this scheme, the channel is estimated based on the received data and the knowledge of training symbols during training symbol transmit. Then, the acquired knowledge of the channel is used for data detection. This method relies upon the insertion of known phasors into the stream of useful information symbols for the purpose of channel sounding. These pilot symbols allow the receiver to extract channel attenuations and phase rotation estimates for each received symbol, facilitating the compensation of fading envelope and phase. The optimal choice of training signals is usually investigated by minimizing mean square error (MSE) of the linear MIMO channel estimator.

In BCE techniques, the use of pilot (training) symbols that consume valuable channel capacity is avoided, but instead the channel is estimated by employing inherent information in the received signals as well as the transmitted signals. Blind algorithms typically require longer data records and higher complexity. SBCE schemes use a few training symbols to provide the initial MIMO channel estimation and exchange the information between channel estimator and the data detector iteratively. In this technique, information about the unknown received signals is used for channel estimation purpose. In DDCE techniques, both the pilot symbols as well as the re-modulated detected message symbols are employed for channel estimation and this scheme provide a more reliable channel estimate method counterpart. By using these techniques, the number of pilot symbols being used for channel estimation could be drastically reduced. 
International Journal of Computer Networks \& Communications (IJCNC) Vol.3, No.5, Sep 2011

In this paper, TBCE scheme for MIMO channel is modeled and simulated. Our goal is to compare the performance of the Least Square (LS), Linear Minimum Least Square Error (LMMSE), EKF, and IEKF channel estimators in the Rayleigh flat fading MIMO channel. The estimation of Rayleigh flat fading MIMO channel at pilot frequencies for comb type can be based on the adaptive filters [8] including LS, LMMSE or Least-Mean-Square (LMS), EKF [9$11]$ and IEKF. Simulation results show that IEKF scheme achieves best BER performance in comparison with LS, MMSE and EKF.

\section{SySTEM MODEL}

Consider a MIMO system equipped with transmit antennas and receive antennas. The block diagram of baseband MIMO-OFDM system is shown in Figure 1.

The multiple transmitting and receiving antennas can be employed with OFDM to enhance the communication capacity and quality of mobile wireless Systems. MIMO is known to boost capacity. In the case of high data-rate transmission, the multipath nature of the communication environment causes the MIMO channels to become frequency-selective. However, OFDM transmission scheme can convert such frequency-selective MIMO channels into an array of parallel frequency-flat MIMO channels by which the receiver complexity is drastically reduced. The combination of these two powerful techniques, MIMO and OFDM to form Multiple Input Multiple Output-Orthogonal Frequency Division Multiplexing (MIMO-OFDM) Systems, is very attractive, and is considered one of the most promising solutions to improve the signal rate of broadband wireless communication Systems.

The binary information is first grouped, coded, and mapped according to the modulation in a "modulation". After the modulation, guard band and cyclic prefix are inserted to the given data stream where the cyclic extension of time length $\mathrm{T}_{\mathrm{G}}$, chosen to be larger than the expected delay spread is to avoid inter symbol and inter carrier interferences. This is divided into symbols (which contain some part of the stream), according to the number of transmitted antennas. For the symbols Inverse Fast Fourier Transform (IFFT) block transforms the data sequence into time domain. The channel is modeled as the random noise channel.

Receiver, after passing through FFT block, it transforms the data back to frequency domain. After channel estimation and STBC decoding, the guard band and cyclic prefix is removed. Lastly, the binary information data is obtained back after the demodulation.

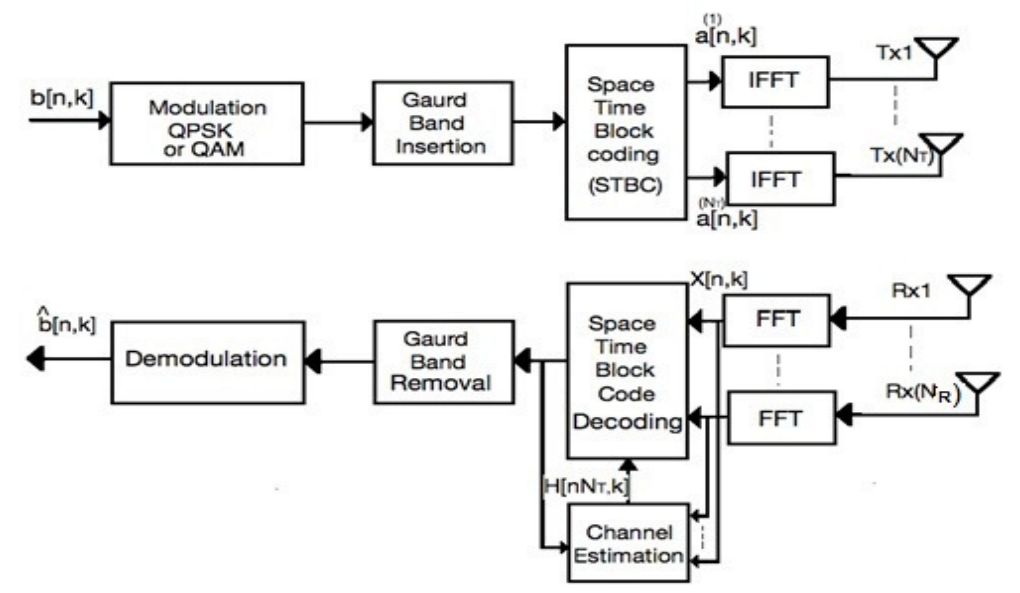

Figure 1. A Digital Implementation of a Baseband MIMO-OFDM system 
International Journal of Computer Networks \& Communications (IJCNC) Vol.3, No.5, Sep 2011

As Figure 1 shows, we use $\mathrm{N}_{\mathrm{T}}$ transmit antennas, $\mathrm{N}_{\mathrm{R}}$ receive antennas, $n$ OFDM symbols and $K$ subcarriers in a MIMO-OFDM system.

The transmitted symbol vector is given as

$a[n, k]=\left[a^{(1)}[n . k] \ldots . \quad a^{\left(N_{T}\right)}[n . k]\right]^{T}, \mathrm{n} \in Z, \mathrm{k}=0 \ldots \ldots \mathrm{K}-1$, where $a^{(i)}[n . k]$ indicates the symbol transmitted at the symbol time $n$, subcarrier $k$, and antenna $i$. The $n$th OFDM symbol $S_{n}[m]$ can be acquired by performing an inverse fast discrete Fourier transform (IFFT) to the $a[n, k]$ and inserting a $C P$ of length $L_{C P}$

$$
S_{n}[m]=\left\{\begin{array}{c}
\frac{1}{\sqrt{K N_{T}}} \sum_{k=0}^{K-1} a[n, k] e^{j 2 \pi m k / K}, m=-L_{C P} \ldots \mathrm{K}-1 \\
0, \quad \text { else }
\end{array}\right.
$$

Thus the duration of each OFDM symbol is $c p N=K+L_{C P}$. The overall baseband transmitted signal is

$$
\mathrm{S}[\mathrm{m}]=\sum_{n=-\infty}^{+\infty} S_{n}[m-n N]
$$

The signal from each receiver is formed by the parameter matrix $\boldsymbol{H}[m, l]$ of the fading MIMO $\mathrm{N}_{\mathrm{T}} \times \mathrm{N}_{\mathrm{R}}$ channel [11], the transmitted signal $\boldsymbol{S}[\mathrm{m}]$, and the noise $\boldsymbol{\eta}[\mathrm{m}] . \boldsymbol{\eta}[\mathrm{m}]$ is stationary white Gaussian noise which distribution is expressed by $\mathrm{N}\left(0, \sigma_{\eta}^{2}\right)$. Thus the received signal can be expressed as follows,

$$
S_{n}[m]=\left\{\begin{array}{c}
\frac{1}{\sqrt{K N_{T}}} \sum_{k=0}^{K-1} a[n, k] e^{j 2 \pi m k / K}, m=-L_{C P} \ldots \mathrm{K}-1 \\
0, \quad \text { else }
\end{array}\right.
$$

The receiver signal $\boldsymbol{r}[\mathrm{m}]$ is demodulated by removing cyclic prefix and performing fast Fourier transform (FFT).

$$
X[n, k]=\frac{1}{\sqrt{K}} \sum_{m=0}^{K-1} r[n N+m] e^{-j 2 k m / K}
$$

If $N f_{\text {Doppler }}<<1$ and $H[m, l]=h_{l}[n] \quad($ Here $\mathrm{n}=\mathrm{m})$ varies negligibly within one OFDM symbol, the input/output relation can be expressed as below,

$$
X[n, k]=\widehat{H}[n . k] a[n . k]+\hat{\eta}[n . k]
$$

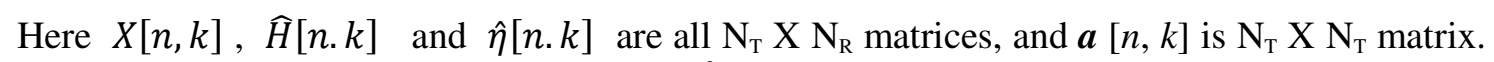
The information regarding channel matrix $\widehat{H}[n . k]$ can be obtained by the channel estimation method based on EKF and IEKF which is described as follows.

\section{Pilot ArRangements}

Use of pilot symbols for channel estimation introduces overhead and it is desirable to keep the number of pilot symbols as minimum as possible. The number of pilot tones necessary to sample the transfer function can be determined on the basis of sampling theorem. The frequency domain channel's transfer function $H$ (f) is the Fourier transform of the impulse response $\mathrm{h}(\mathrm{t})$. Each of the impulses in the impulse response will result a complex exponential function $\mathrm{e}^{-\mathrm{j} 2 \pi \tau \mathrm{T}_{\mathrm{s}}}$ in the frequency domain, depending on its time delay $\tau$, where $\mathrm{Ts}_{\mathrm{s}}$ is the symbol time. In order to sample this contribution to $\mathrm{H}$ (f) according to the sampling theorem, the maximum pilot spacing $\Delta \mathrm{p}$ in the OFDM symbol is

$$
\Delta p \leq \frac{N}{2 \mathcal{T} / T_{S}} \Delta f
$$


International Journal of Computer Networks \& Communications (IJCNC) Vol.3, No.5, Sep 2011

where $\Delta \mathrm{f}$ is the subcarrier bandwidth

Either inserting pilot tones into all of the subcarriers of OFDM symbols with a specific period or inserting pilot tones into each OFDM symbol can perform the channel estimation. The first one, block type pilot channel estimation, has been developed under the assumption of slow fading channel. This type of pilot arrangements works well when the channel transfer function is not changing very rapidly. The later one, comb type pilot arrangement, can be used easily for tracking fast channels. In comb arrangements, every OFDM symbol has some pilot tones, therefore these type of patterns works well in highly varying environments.

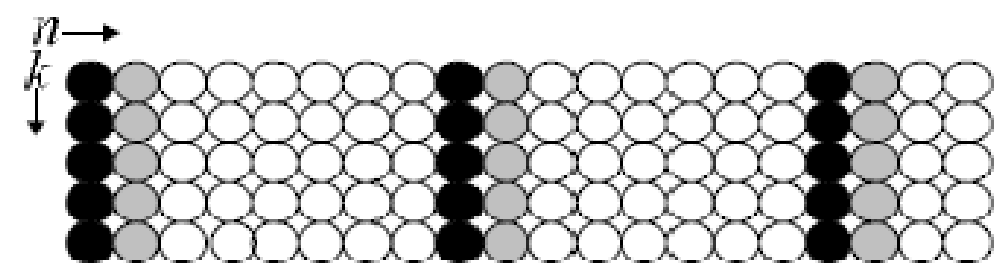

Figure 2. Pilot structure in each antenna

For a $2 \times 2$ MIMO-OFDM system, the pilot sequences are inserted as Figure 2 shows, i.e; $P_{1}=[1,1]^{T}, . P_{2}=[1,-1]^{T}$.

\subsection{Alamouti Space- Time Coding}

In 1996, Alamouti proposed the first space-coding for a MIMO 2x 2 system. The proposed matrix is as follows:

$$
C_{2}=\left[\begin{array}{cc}
c_{1} & c_{2} \\
-c_{2}^{*} & c_{1}^{*}
\end{array}\right]
$$

where * denotes complex conjugate.

It takes two time-slots to transmit two symbols. Using the optimal decoding scheme discussed below, the bit-error rate (BER) of this STBC is equivalent to $2 \mathrm{nR}$-branch maximal ratio combining (MRC). This is a result of the perfect orthogonality between the symbols after receive processing (i.e ;), there are two copies of each symbol transmitted and ' $\mathrm{nR}$ ' copies received. This is a very special STBC. That is to say that it is the only STBC that can achieve its full diversity gain without needing to sacrifice its data rate.

\section{Channel Estimation}

In wireless communication systems, the time-varying nature of the channel as well as its frequency selectivity in a multipath scenario is considered as one of the major challenges. For accurate transmitted signal demodulation, equalization, decoding, and a host of other baseband processing applications, the provision of perfect and up to date channel knowledge is very vital. Consequently, channel estimation remains an important block in the signal processing stages at the receiver of both the existing and the evolving wireless communication systems. The key to fully utilize the MIMO capacity relies heavily on the requirement of accurate channel estimation. For block type arrangements, channel at pilot tones can be estimated by using LS or LMMSE estimation, and assumes that channel remains the same for the entire block. 
International Journal of Computer Networks \& Communications (IJCNC) Vol.3, No.5, Sep 2011

LMMSE estimation has been shown to yield 10-12 dB gain in SNR over LS estimation for same mean square error of channel estimation. A low rank approximation is applied to linear MMSE by using frequency correlations of the channel to eliminate major drawback of MMSE, namely complexity. Comb type pilot tone estimation, has been introduced to satisfy the need for equalizing when the channel change seven in one OFDM block. The comb-type pilot estimation consists of algorithms to estimate the channel at pilot frequencies and to interpolate the channel. The estimation of channel at pilot frequencies for comb type based channel estimation can be based on LS [8], LMMSE or LMS. MMSE has been shown to perform much better than LS.

\subsection{LEAST SQUARE ESTIMATION}

The idea behind least squares is to fit a model to measurements in such a way that weighted errors between the measurements and the model are minimized. The LS estimate of the attenuations $\mathrm{h}$, given the received data $\mathrm{Y}$ and the transmitted symbols $\mathrm{X}$ is

$$
\widehat{h}_{l s}=X^{-1} Y=\left[\frac{y_{0}}{x_{0}} \frac{y_{1}}{x_{1}} \ldots \frac{y_{N-1}}{x_{N-1}}\right]^{T}
$$

"Least squares" means that the overall solution minimizes the sum of the squares of the errors made in solving every single equation. The objective consists of adjusting the parameters of a model function to best fit a data set. A simple data set consists of $n$ points (data pairs) ( $x_{i}$, $\left.y_{i}\right), i=1, \ldots, n$, where $x_{i}$ is an independent variable and $\mathrm{y}_{\mathrm{i}}$ is a dependent variable whose value is found by observation. The model function has the form $\mathrm{f}(\mathrm{x}, \beta)$, where the $m$ adjustable parameters are held in the vector $\beta$. The least squares method finds its optimum when the sum, $S$, of squared residuals is a minimum.

$$
S=\sum_{i=1}^{n} r_{i}^{2}
$$

A residual is defined as the difference between the value predicted by the model and the actual value of the dependent variable

$$
r_{i}=y_{i}-f\left(x_{i}, \beta\right)
$$

\subsection{Minimum Mean SQUARE ERror ESTIMATION}

LMMSE estimate has been shown to be better than the LS estimate for channel estimation in OFDM systems based on block type pilot arrangement. Regarding the mean square error estimation, the LMMSE estimate has about 10-15dB gain in SNR over LS estimate for same MSE values. The major drawback of the LMMSE estimate is its high complexity, which grows exponentially with observation samples. Assume that all the available LS estimates are arranged in a vector $\widehat{p}$ and the channel values that have to be estimated from $\widehat{p}$ are in a vector $\mathrm{h}$. The channel estimation problem is now to find the channel estimates $\widehat{\mathrm{h}}$ as a linear combination of pilot LS estimates $\widehat{p}$. The minimum mean square error estimate for this problem is given by

$$
\hat{h}_{l m s e}=R_{h \hat{p}}\left(R_{p \hat{p}}\right)^{-1} \hat{p}
$$

$R_{h \hat{p}}$ is the cross-covariance matrix between $\mathrm{h}$ and the noisy pilot estimates $\hat{\mathrm{p}}$, given by

$$
R_{h \hat{p}}=E\left\{h \hat{p}^{H}\right\}
$$


International Journal of Computer Networks \& Communications (IJCNC) Vol.3, No.5, Sep 2011

$R_{p \hat{p}}$ is the auto-covariance matrix of the pilot estimates, and is given by

$$
\begin{aligned}
R_{p \hat{p}} & =E\left\{\hat{p} \hat{p}^{H}\right\} \\
& =R_{p p}+\sigma_{n}^{2}\left(p p^{H}\right)^{-1}
\end{aligned}
$$

where $\sigma_{\mathrm{n}}^{2}$ is the variance of additive channel noise. The superscript $(\cdot)^{\mathrm{H}}$ denotes Hermitian transpose.

Assuming the same signal constellation on all tones and equal probability on all constellation points, we have $\mathrm{E}\left\{\left(\mathrm{pp}^{\mathrm{H}}\right)^{-1}\right\}=\mathrm{E}\left\{\left|\frac{1}{p_{k}}\right|^{2}\right\} \mathrm{I}$, where I is the identity matrix. Defining the average signal-to-noise ratio as

$$
S N R=E\left\{\left|p_{k}\right|^{2}\right\} / \sigma_{n}^{2}
$$

we obtain a simplified estimator,

$$
\hat{h}_{l m m s e}=\mathrm{R}_{\mathrm{hh}}\left(R_{h h}+\frac{\beta}{S N R} I\right)^{-1} \hat{p}
$$

\subsection{EKF AND IEKF FORMULATIONS}

\subsubsection{EKF BASED MODELING}

Kalman filter is a recursive predictive filter based on the use of state space techniques and recursive algorithms. It demands the description of the dynamical problem in a state-space form which includes a system model and an observation model which is considered only for linear systems but there may be existence of non-linear systems. One approach for such non-linear channel issues is so-called Extended Kalman filter.

Extended Kalman filter extend the use of Kalman filter through linearization procedure.

Consider linear dynamic system whose state equation is given as [12-13]

$$
x_{k}=F_{k-1} x_{k-1}+w_{k-1}
$$

Where F- Process equation $\& w_{k-1}$ - Process Noise with zero mean and known co-variance matrix $Q_{k}$

The output equation is given as

$$
Z_{k}=H_{k} x_{k}+v_{k}
$$

Where $\mathrm{H}=$ Measurement Equation \& v-Measurement Noise with zero mean and known covariance matrix $R_{k}$

The Predicted State Estimated Equation of EKF are given as [14-15]

$$
\begin{gathered}
x_{k \mid k-1}=F_{k-1} x_{k-1 \mid k-1} \\
P_{k \mid k-1}=F_{k-1} P_{k-1 \mid k-1} F_{k-1}^{T}+Q_{k-1}
\end{gathered}
$$

The Corrected State Estimated Equations of EKF are given as

$$
\begin{gathered}
K_{k}=H_{k} P_{k \mid k-1} /\left(H_{k} * H_{k}^{T} * P_{k \mid k-1}+R_{k}\right) \\
P_{k \mid k}=P_{k \mid k-1}\left(1-H_{k} K_{k}\right) \\
x_{k \mid k}=x_{k \mid k-1}+K_{k}\left(Z_{k}-H_{k} x_{k \mid k-1}\right)
\end{gathered}
$$


International Journal of Computer Networks \& Communications (IJCNC) Vol.3, No.5, Sep 2011

where $K_{k}$ is the Kalman gain

\subsubsection{IEKF BASED MODELING}

As the BER performance given by Extended Kalman filter is not satisfactory So, Iterative Extended Kalman Filter is implemented to get better BER performance.

The state equation is given as

$$
x_{k}=F_{k-1} x_{k-1}+w_{k-1}
$$

where F- Process equation $\& w_{k-1}$ - Process Noise with zero mean and known co-variance matrix $Q_{k}$

The output equation is given as

$$
Z_{k}=H_{k} x_{k}+v_{k}
$$

Where $\mathrm{H}=$ Measurement Equation \& v-Measurement Noise with zero mean and known covariance matrix $R_{k}$

The Predicted State Estimated Equation of IEKF are given as

$$
\begin{gathered}
x_{k \mid k-1}=F_{k-1} x_{k-1 \mid k-1} \\
P_{k \mid k-1}=F_{k-1} P_{k-1 \mid k-1} F_{k-1}^{T}+Q_{k-1}
\end{gathered}
$$

The Corrected State Estimated Equations of IEKF are given as

$$
\begin{array}{r}
K_{k}=H_{k}^{T} P_{k \mid k-1} x_{k \mid k} /\left(H_{k} * H_{k}^{T} * P_{k \mid k-1} x_{k \mid k}+R_{k}\right) \\
P_{k \mid k}=P_{k \mid k-1}\left(1-H_{k} K_{k} x_{k \mid k}\right) \\
x_{k \mid k}=x_{k \mid k-1}+K_{k}\left(Z_{k}-h x_{k \mid k-1}+H_{k} x_{k \mid k}\left[x_{k \mid k-1}-x_{k \mid k}\right]\right)
\end{array}
$$

where $K_{k}$ is the Kalman gain

\section{Simulation ReSUlts AND Discussions}

In this section simulation results are presented and discussed in a bit to characterize the achievable performance of the Iterative Extended Kalman Filter scheme in comparison with Least Squares (LS), Minimum Mean Square Error (MMSE) and Extended Kalman Filter (EKF). A QPSK-modulated OFDM system with $\mathrm{K}=64$ subcarriers and a total bandwidth of $6 \mathrm{MHz}$ is assumed. The symbol duration, $T s$ is $80 \mu \mathrm{s}$, while CP length is 16 samples $(1 / 4$ of the symbol period) with the CP period, $T_{g}=20 \mu$ s. Consequently, the total block period, $T$ is $100 \mu \mathrm{s}$. The space-time coding scheme is Alamouti's STBC with $1 / 2$ rate and the decoding scheme used is Maximum likelihood (ML) technique with only linear processing. The graphs are plotted for Bit Error Rate versus SNR and Mean Square Error versus SNR by taking 2x2 MIMO-OFDM system. The number of OFDM symbols considered here are 8 . These results infer that Iterative Extended Kalman Filter gives better performance than the other channel estimation techniques. 
International Journal of Computer Networks \& Communications (IJCNC) Vol.3, No.5, Sep 2011

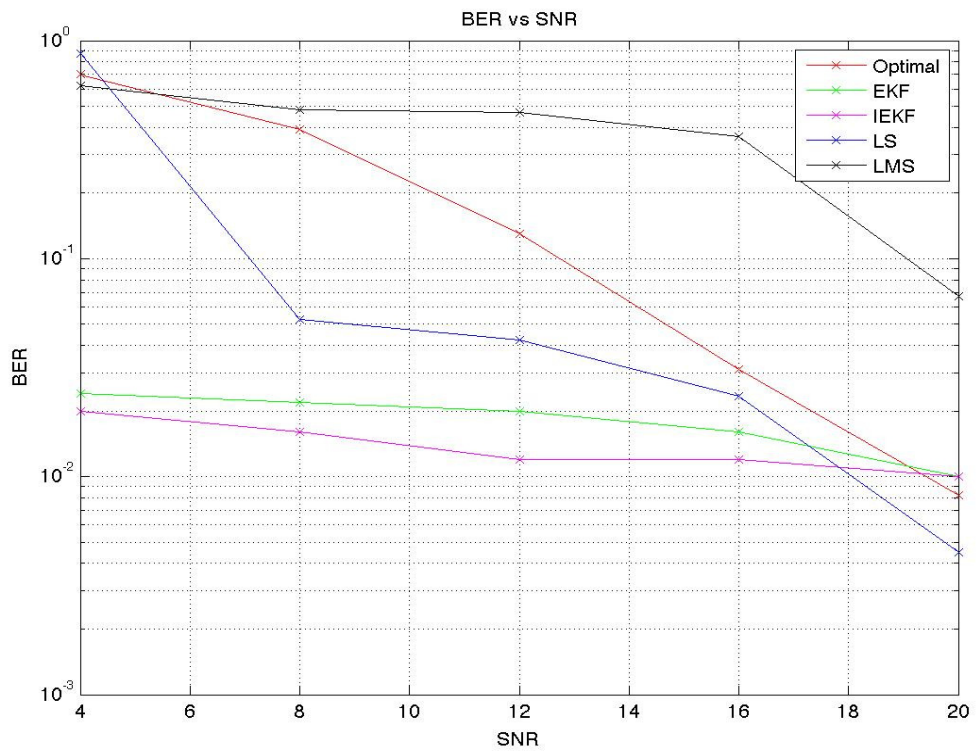

Figure 3. Comparative BER exhibited by the Optimal, LS, MMSE, EKF and IEKF channel estimators

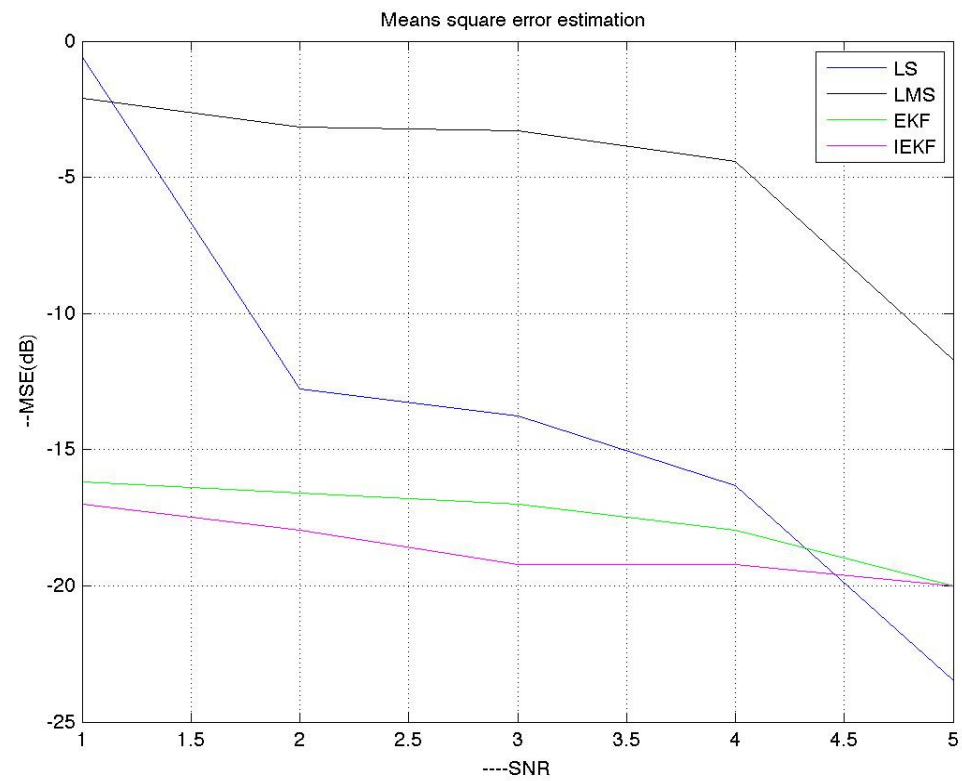

Figure 4.Comparative MSE exhibited by the LS, MMSE, EKF and IEKF channel estimators

\section{Conclusion}

In this paper the channel estimation for MIMO System in combination with Orthogonal Frequency Division Multiplexing transmission scheme is addressed. Having reviewed previous contributions in this area, we proposed computationally efficient and robust channel estimation schemes for MIMO-OFDM systems. This method exploits pilot symbol and the IEKF 
International Journal of Computer Networks \& Communications (IJCNC) Vol.3, No.5, Sep 2011

algorithm to estimate channel without any prior statistical knowledge of channel or noise. Simulation results demonstrate that the proposed IEKF method has moderate computational complexity and higher performance than the LMS, the LS and the EKF methods. According to non-linear or linear characteristics of channels we can select reasonable channel estimation method for MIMO-OFDM systems. However, we believe that there are quite a lot of research works that need to be carried out especially for MIMO-OFDM communication Systems. This is because it is of common knowledge that MIMO-OFDM technique will have a crucial role to play in the implementation of the future generation of mobile wireless Systems, especially the fourth generation $(4 \mathrm{G})$ and fifth generation $(5 \mathrm{G})$ Systems and even higher generation that could be conceived.

\section{REFERENCES}

[1] G. J. Foschini, M. J. Gans, "On limits of Wireless Communications in a fading environment when using multiple antennas",Wireless Personal. Communication, Mar.1998, 6: pp. 331-335.

[2] I Hen, MIMO architecture for Wireless Communications, Vol.10, No.2, Mobility Group, Intel. Corporation; 2006.

[3] S. M. Alamouti, "A simple transmit diversity technique for Wireless Communications", IEEE Journal on Select. Areas Communications, Oct. 1998, 16(8):pp.1451-1458.

[4] V. Tarokh, H. Jafarkhani, A R Calderbank, "Space-time block codes from orthogonal designs", IEEE Trans. Information. Theory, July 1999, 45(5):pp.1456-1467.

[5] G. L. Stuber, J. R. Barry, S. W. McLaughlin, Ye Li, M. A.Ingram, T. G. Pratt, "Broadband MIMOOFDM Wireless Communications", Proceedings of the IEEE, Feb 2004, 92 (2).

[6] Ye Li, N. Seshadri, S. Ariyavisitakul, "Channel estimation for OFDM systems with transmitter diversity in mobile wireless channels", IEEE Journal on selected Areas in Communications, March 1999, 17(3):461-471.

[7] Ye Li, "Simplified channel estimation for OFDM systems with multiple transmit antennas", IEEE Trans. Wireless Communications.Vol.1, pp.67-75, Jan. 2002.

[8] S. Haykin, Adaptive Filter Theory (Fourth Edition), Publishing House of Electronics Industry, Beijing, 2003.

[9] D. Schafhuber, M. Rupp, G. Matz, F. Hlawatsch, "Adaptive identification and tracking of doubly selective fading channels for wireless MIMO-OFDM systems", 4th IEEE Workshop on SPAWC, June 2003, pp.417- 421.

[10] Zhiqiang Liu, Xiaoli Ma, G.B.Giannakis, "Space-time coding and Kalman filtering for timeselective fading channels", IEEE Trans. on Communications, Feb 2002, 50(2): pp.183-186.

[11] D. Schafhuber, G. Matz, F. Hlawatsch, "Kalman tracking of time-varying channels in Wireless MIMO-OFDM systems", the Thirty-Seventh Asilomar Conference on Signals, Systems and Computers, Nov. 2003, 2: pp.1261-1265.

[12] Yongming Liang, Hanwen Luo, Youyun Xu, Jianguo Huang "Channel Estimation Based on Extended Kalman Filtering for MIMO-OFDM Systems", Proceedings of the IEEE 2006.

[13] Flavio Celso Trigo, Raul Gonzalez-Lima "Iterated Extended Kalman Filter with Adaptive State Noise Estimation for Electrical Impedance Tomography", Technology Meets Surgery International in 2005 by $\mathrm{ABCM}$. 
International Journal of Computer Networks \& Communications (IJCNC) Vol.3, No.5, Sep 2011

[14] K. Myers, B. Tapley, "Adaptive sequential estimation with unknown noise statistics", IEEE Transactions on Automatic Control, Aug 1976.Volume 21(4):520-523.

[15] R. Mehra, "On the identification of variances and adaptive Kalman filtering", IEEE Transactions on Automatic Control, Apr 1970, 15(2):175-184.

\section{Authors}

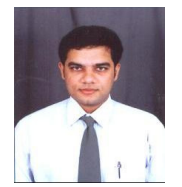

Mr. K. Rajendra Prasad, Assistant Professor, School of Electrical sciences, Department of ECE, K L University, Andhra Pradesh, India. He received B.Tech, M.Tech in ECE, Communication and Radar systems from JNTUH and ANU respectively. Currently he is pursuing Ph.D from JNTU Hyderabad. $\mathrm{He}$ is the faculty member in signal processing research group, K L University. He is interested to work in the research topics related to Multicarrier signal processing, MIMO-OFDM systems and Target tracking algorithms.

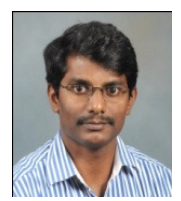

Dr.M.Srinivasan, Professor, currently associated with Meerut Institute of Engineering and Technology, Meerut, U.P, India. He received B.E in Instrumentation and Control Engineering (Madras University, India) M.E in Control System Engineering, PhD in Engineering from Jadavpur University, India respectively. He is Member in IEEE, AIAA, Associate Member; IE (India). Earlier he associated with signal processing research group as group head and established the Centre for Intelligent Systems in K L University, India. His area of interests includes Target tracking, Statistical estimations and detection, and Post Kalman filtering techniques. He authored nearly 20 research papers in various reputed journals and conferences. 